\title{
Development of a Mobile Multi-device Nutrition Logger
}

\author{
Andreas Seiderer \\ University of Augsburg \\ Augsburg, Germany \\ seiderer@hcm-lab.de
}

\author{
Simon Flutura \\ University of Augsburg \\ Augsburg, Germany \\ flutura@hcm-lab.de
}

\author{
Elisabeth André \\ University of Augsburg \\ Augsburg, Germany \\ andre@hcm-lab.de
}

\begin{abstract}
In this paper we present a mobile system for nutrition logging which integrates multiple devices and modalities to facilitate food and drink tracking. The user is free to decide in each situation to use the most appropriate device combination out of a smartphone, smartwatch and smartscale. We describe the design and implementation of our system which is based on a requirements analysis. Finally, first results of a preliminary in-situ study with the prototype are reported giving first hints about the benefits and challenges of this multi-device approach in daily life scenarios.
\end{abstract}

\section{CCS CONCEPTS}

- Human-centered computing $\rightarrow$ Mobile devices; User interface programming;

\section{KEYWORDS}

Nutrition Logging, Food Journal, Multi-Device, Smartwatch

\section{ACM Reference Format:}

Andreas Seiderer, Simon Flutura, and Elisabeth André. 2017. Development of a Mobile Multi-device Nutrition Logger. In Proceedings of 2nd ACM SIGCHI International Workshop on Multisensory Approaches to Human-Food Interaction (MHFI'17). ACM, New York, NY, USA, 8 pages. https://doi.org/10. $1145 / 3141788.3141790$

\section{INTRODUCTION}

In general nutrition is a complex topic where different types of questions occur that can often only be answered by logging the intake of foodstuff. This can be especially helpful for people suffering from diseases like diabetes, overweight and food allergies, but also for others that wish to detect nutrient deficiencies or a too high calorie intake prematurely.

Nevertheless, recording the exact type and amount of each food and drink is a time-consuming task and often makes people to stop it soon as they find the effort being too high [7]. In this paper we will present a multi-device system consisting of a smartphone, a smartwatch and a smartscale. We will show how to leverage the situation-specific capabilities of the individual devices in order to reduce the overall effort involved in the nutrition logging process.

The smartphone provides the most flexible user input possibilities and already significantly reduces the effort in comparison to

(C) Author ACM 2017. This is the author's version of the work. It is posted here for your personal use. Not for redistribution. The definitive Version of Record can be found at

https://doi.org/10.1145/3141788.3141790 traditional pen and paper methods [15]. A smartwatch can supplement a smartphone since it is always in reach and can be used in a less conspicuous manner. However, due to the small screen size, it is limited in its input capabilities. A smartwatch is in particular useful in situations when a user is in a hurry and when repeating foodstuff has to be logged that can directly be selected from favorites or recently used entries. An entry with missing data like the amount is better than no record at all since the user still can edit and complete the entry with the smartphone afterwards. Already incomplete entries can provide medical relevant information about the time and frequency of nutrition intake [15].

Usually it is necessary to input the amount of food and drink intake. Estimating it can be a hard task. We therefore include a Bluetooth scale prototype (smartscale) in the system. The scale is designed to be easy to use by transmitting the current weight to the smartphone and the smartwatch without requiring to pair them. The user can leave the scale switched on for several weeks without charging. Furthermore, we tried to make the scale compact so that it is possible to place it wherever the user prefers to use it. Like with the smartwatch the usage of this device is optional.

While the usage of multiple devices appears to be a promising option for food tracking, the design of multi-device user interfaces that enable smooth transitions from one device to the other is a non-trivial task. To our knowledge this paper contains the first multi-device application combining smartwatch and scale with a smartphone. In literature covering multi-device interaction Dong et al. [8] identified two dimensions of inter-device usability. Users should be able to transfer knowledge obtained from the usage of a previous device to the next one (knowledge continuity) and they should be able to continue a task started on one device on another one (task continuity). Both types of continuity may be supported by a consistent user interface design to enable the user to easily switch from one device to the other. Another aspect to consider in the design of multi-device user interfaces is the timing of the tasks to be carried out by the user. Sørensen et al. [21] distinguish between the simultaneous and sequential execution of tasks distributed over multiple devices. In the case of food logging, a user may place an item on a smartscale while adding information on the type of food via his or her smartphone (simultaneous use). Furthermore, the user may want to use the smartphone to complement information on food consumption recorded with the smartwatch while being on route before (sequential usage). In both cases, automatically recorded or manually input information on the foodstuff has to be synchronized across devices.

With our prototype we want to find answers to the questions:

- Do people make use of multiple devices and if so, how are they used?

- On which factors does the device usage depend?

- When are users doing the nutrition logging? 
In the next section, we will discuss related work on recognition techniques and user interfaces to support (semi-)automated nutrition logging. After that, we will identify requirements for a multi-device nutrition logging system. We then describe how the requirements have been met by the design and implementation of a system integrating a smartphone, a smartwatch and a smartscale. To evaluate our multi-device approach to food logging, we conducted preliminary in-situ studies whose results will be discussed and can be used for further in-depth studies and extensions.

\section{RELATED WORK}

\subsection{Logging of Nutrition Intake}

The simplest form of nutrition logging is the usage of pen and paper which requires the biggest recording effort, however. Especially the estimation of the amount of foodstuff is a big problem. For food there are several manual portion size estimation aids (PSEA) that enable a higher accuracy than photos, but they can still introduce high inaccuracies depending on the individual [21].

For an automatic detection of the nutrition intake cycle, several processes can be recognized. Augmented cutlery, such as a fork [13] or an augmented drink vessel [4], can be used as sensors. Pressure changes that occur during the manipulation of plates or vessels on a table can be detected using a pressure sensitive table cloth [25]. To recognize arm movement leading a piece of food or a drink to the mouth, accelerometers have been used [1] which can be included in a smartwatch [24]. Chewing can be sensed by detecting the vibrations using audio [2]. Microphones of a regular headset [11] or smartwatch [14] are applicable, too.

Automatic methods that are able to detect the intake of food and / or drink mostly can't be used to detect the type and / or amount of the foodstuff. For example with audio data it is possible to discern food according to its consistency [23] so that for instance soft or crispy food can be distinguished. Nevertheless, with audio data, it is not possible to detect whether a person ate fatty potato chips or fat reduced ones (with the same consistency). Most precise methods are the ones that can directly weigh the foodstuff like a modified vessel for a drink. Relatively good precision can also be achieved by camera-based volume estimations [12] in case the food type has also been detected correctly.

Our current prototype supported by a smartscale is work in progress and currently serves as a basis for further automation of the nutrition logging process. Nevertheless, currently the limitations identified above indicate that manual or partly automated systems for nutrition intake logging are still required if a higher accuracy and precision for food recordings is important. It might even be beneficial to involve the user in the manual completion of the tracked data to make him or her aware of possible misbehavior $[6,15]$.

\subsection{Smartphone and Smartwatch Apps}

Mobile devices offer various methods to simplify the logging process and are nearly always at reach. The preference of a smartphone app over a website or paper diary has been the result of a study conducted by Carter et al. [5] where just users of the smartphone app still recorded their daily nutrition intake after six months. To get an impression of features offered by popular smartphone apps, we refer to a survey of nutrient-related mobile apps by Franco et al. [10] and an overview of related health apps by Murnane et al. [20].

An app that tries to simplify nutrition intake logging by using a multi-modal approach including image recognition, but also text input as a fallback is presented by Lim et al. [17]. Their study participants were trying the automatic detection occasionally, but in the end they preferred manual input as it was more reliable although taking photos was more convenient. This observation indicates that as long as automatic systems don't work nearly as precisely as manual logging they have to be designed in a way that manual input for correction or entry is possible.

Since smartwatches are still not widely used, just few apps and relatively few related work can be found dealing with the task of nutrition logging. Arsand et al. [3] presented a smartphone and smartwatch app (for Pebble) for diabetes patients that act as a diary. The apps are optimized for this target group, but it was found that the smartwatch simplified the entering process which should also be the case for nutrition logging.

Lutze et al. [18] presented a special smartwatch app for elderly people that serves to prevent dehydration and to provide support in emergency situations. In their case the smartwatch was mainly used standalone, providing sensor data, giving reminders to drink and allowing the user to answer simple questions in case an emergency situation was assumed by the system before help is being called. Also in their work it becomes apparent that the smartwatch offers the advantage of allowing fast input since it is in easy reach whenever there is a need to interact with it.

According to the related work the usage of a smartphone is beneficial as they are widely spread and nearly always at reach. Smartwatches show additional advantages for example in situations where fast input is necessary as it is possible to directly interact with them as they are in direct reach.

\subsection{Smartscale}

In the following we define a smartscale as a scale that can directly communicate with a smartphone or smartwatch to enable easy weight transmission.

SITU $^{1}$ is a commercial smartscale intended to be used with an iOS exclusive app that can estimate the calories and nutrients by the weight of food after inputting its type. Our most recent scale prototype is more compact than the SITU scale and doesn't require to be paired with a smartphone. We also wanted to investigate the usage in combination with a smartphone and smartwatch without any restrictions by proprietary software.

Recently methods show how specific smartphones can be used directly as a scale to weigh smaller objects that are being placed on their screen by exploiting pressure sensitive touch features ${ }^{2}$. Although this would have been an interesting option, currently this feature is very device-specific, it is not as precise as a scale, it can just be used with small foodstuff / vessels that fit on the screen and the maximum weight is quite limited. This is why we chose to focus on smartscales. Smartscales are already used to weigh food and drink and usually provide a good precision especially compared

\footnotetext{
${ }^{1}$ http://situscale.com/ (accessed: July 27 2017)

${ }^{2}$ http://money.cnn.com/2015/09/03/technology/force-touch-weigh-objects-huawei/ (accessed: July 27 2017)
} 
to automatic detection methods or often also estimations by users. For a nutrition logging system they offer a helpful addition and simplify the entry of the amount of foodstuff.

Lessel et al. [16] used a smartscale as a drink sensor for an app providing gamifications to motivate users to drink more. Soubam et al. [22] presented a system that incorporates a smartwatch to detect drinking activity and a smartscale to measure the fluid amount. The prototypes bear some similarities to ours since there are not many design options when a load cell is being used as sensor. The prototype by Lessel et al. has a top plate that is surrounded by a rim of the case which prevents directly positioning larger plates on the scale. Additionally, it is necessary to press a button located on the scale to send the weight to the smartphone app and may get hidden by a plate. The smartscale by Soubam et al. partly lacks a surrounding case and it is unclear whether the battery or a charger is integrated.

The main difference of our prototype is the communication method between the smartphone and smartscale by using BLE advertisement packets which makes pairing unnecessary and allows sharing one scale with multiple users. By using this transmission method and techniques to reduce power consumption, the battery of our prototype usually lasts for three weeks.

\section{REQUIREMENTS}

In this section we will present the most important requirements we gathered for our nutrition logging system from the following sources: related work, popular Android apps (PA) and semi-structured interviews (I). We followed these requirements during the development of our prototype, and we will also discuss to what extent they were met later on. Several requirements were derived from the work by Cordeiro et al. [7], which points out many problems that may occur during food journaling. They gathered a variety of issues by compiling the results of a survey of 141 current and lapsed food journaling persons and additionally collecting posts from the community forums of three mobile food journals. Furthermore, we considered the overviews of nutrient-related mobile apps by Franco et al. [10] and health apps by Murnane et al. [20].

\subsection{Popular Apps}

We selected four apps by their popularity and feature set from the Android app store to find further requirements: "MyFitnessPal" (1.469.230 ratings), "S Health" (233.598 ratings), "FatSecret" (171.922 ratings) and "Lifesum" (70.224 ratings). Since most of the apps don't just focus on an efficient way to record nutrition intake, but also on statistics, other health related information and tracking of activities, we mainly tested the recording of foodstuff entries. At this time we couldn't find a popular app that includes a smartwatch allowing the input of foodstuff entries, S-Health on the Gear S2 allows for fluid (in counted glasses and cups) only. Each of the apps was used by us for one day to log our nutrition intake. The feature set was examined in detail, containing bar code reading for adding new foodstuff, adjoining topics such as activity recognition and details on healthy nutrition (recommended amount of vitamins). Gamification and visualization approaches were collected, but just considered for future extensions of the prototype.

\subsection{Semi-Structured Interviews}

Finally, we conducted semi-structured interviews with 24 (13 females, 11 males) persons in the age between 16 and 55 (avg. 26.2). Most of them were students of a school or university. All of them were familiar with using a smartphone. The interviews contained about 25 questions concerning demographic data, smartphone usage, experience with health apps, willingness to use smartwatches, privacy concerns, knowledge on tools for nutrition tracking, their motivation to do nutrition logging and preferred schedule for logging. Some questions were reused for the evaluation of the prototype.

\subsection{Reducing the Effort of Nutrition Logging}

The reduction of the overall nutrition logging effort is the main goal of our system. Several requirements have been identified to guide our design towards this target.

- The recording process should be as easy as possible since high logging effort is a "major barrier" [7].

- The system should help determine the amount of a portion since this is usually a hard task [7].

- The system should include a food / drink database to simplify text input (PA).

- User defined foodstuff favorites should be integrated (PA).

- The user should be able to choose from a list of recent foodstuff entries (PA).

- In less public situations, speech recognition could be used as an alternative input method (I).

\subsection{Privacy, Flexibility of Use and Others}

Additionally, following requirements were found that concern the privacy, flexibility of use, reminders and possibility to reflect.

- Adding entries into the system should be possible in an inconspicuous way as some persons don't want to log foodstuff in front of friends or colleagues [7].

- The system should allow the addition of own notes (I).

- The user should be able to edit entries (I).

- People should get reminders since they tend to forget to journal as it has been reported by Kim et al. [15].

- Reflection about intaken foodstuff should be possible [7].

\subsection{Multi-Device Interaction}

Our approach is based on the assumption that the effort required to track food intake may be reduced by leveraging the capabilities of multiple devices. The following requirements have to be met in order to ensure a high amount of inter-device usability:

- In order to enable the user to easily switch from one device to the other, a high amount of GUI consistency has to be maintained across all devices while following standards of the individual platforms [8].

- Data synchronization has to be supported across all devices to enable the synergistic use of multiple devices [21].

The functionality of the system should be split across the different devices to be able to take advantage of their specific strengths [8]. In our case, the smartphone is the main device that includes all features. Due to its small display the smartwatch just shares functionality of the smartphone that is important in situations where fast 
or more private logging is wished. The smartscale can just weigh objects and allows a more comfortable and precise input of the amount of foodstuff while using the smartphone or smartwatch interface.

\subsection{Smartscale Prototype}

The prototype is part of the solution to the requirement to support the recording of the amount of a portion in an easy manner. We decided to include a scale into our nutrition logging system as it is able to measure the weight of food and drink with high precision and its general functionality is already known to users. Even though our prototype is not limited to fluid intake, some requirements identified by Lessel et al. [16] are applicable in our case as well:

- The scale should be easily transportable.

- The stability of the prototype should be high enough to be able to measure foodstuff including a containment.

- Weighing should have a reasonably high precision.

- The prototype should have a relatively low price.

Some additional requirements are not met by the prototype developed by Lessel et al., but are relevant for us:

- It should have very low battery consumption.

- The user should not be bothered with Bluetooth pairing.

- It should be possible to place plates on the scale.

\section{DESIGN OF THE SMARTSCALE}

\subsection{Development Process}

To measure the weight of objects, there are two commonly used sensor types: resistive pressure sensors and load cells. Our first prototype used resistive pressure sensors. This type of sensor is not only more expensive than a load cell, but also suffers from noise and sensor value drift over time. To filter these disturbances out of the signal to obtain a sufficiently precise weight is not a trivial task.

Our second and third prototype are based on load cells that are commonly used in kitchen scales. Quite recently, many tutorials and necessary libraries - especially for Arduinos - can be found on the Internet. Two similar prototypes - also based on load cells have been developed by Lessel et al. [16] and Soubam et al. [22].

Since the scale should be able to communicate with a smartphone energy efficiently, Bluetooth LE is currently the best choice. The requirement for the scale was that the user is able to interact with it just by placing an object on it - usually no further interaction with the scale should be required. Furthermore, no pairing with a smartphone should be necessary. This is why we decided that the scale should use Bluetooth advertisement packets that are broadcasted every two seconds. Bluetooth advertisement packets are also used by Apple's iBeacon or Google's Eddystone protocol. For our prototype, we use custom packets since we send the currently measured weight as payload. Nevertheless, the scale still can be used as a beacon and an app can detect whether the user is near the scale by considering the RSSI value. The broadcasts additionally allow for sharing a scale with multiple persons.

For the second prototype, we modified a low-cost kitchen scale by removing all electronic components except of the load cell and by building an Arduino Mini Pro, a HX711 analog-digital converter
(ADC) and a nRF24L01+ RF module (capable to send Bluetooth compatible packets) into the case. The HX711 ADC was connected to the built-in $5 \mathrm{~kg}$ load cell. Finally, we created a third prototype which is more mobile than the modified kitchen scale since it is smaller (10.0 x $10.0 \times 3.2 \mathrm{~cm}$ compared to $16.2 \times 12.7 \times 3.3 \mathrm{~cm}$ ) and integrates a rechargeable 18650 lithium battery with protection circuit and a capacity of 3.4 Ah. It can be charged with an integrated charger (TP4056) via USB (with adapter cable). The LEDs of the charger indicating the state can be seen from the side. These components are basically the same as used in the work by Lessel et al. [16] and Soubam et al. [22]. We didn't use a case printed by a 3D printer since those are often less stable which has been a problem reported by Lessel and colleagues. For our latest prototype, we use Nordic's NRF51822 Bluetooth 4.0 SoC. This SoC saves space since there is no need to use a microcontroller with an additional Bluetooth module making it more energy efficient. The biggest drawback of the NRF51822 is that there is usually no direct or just limited Arduino support. This is why we first had to port the HX711 library code for this controller to be able to use it directly with Nordic's SDK.

Unlike Soubam et al. we didn't include an accelerometer or gyroscope in our prototypes since we assume that users usually know that a scale should be placed on a stable and even base. Nevertheless, it could be used to detect whether the scale is being transported to save energy. The overall price of our prototypes is about 30 Euro (prototype 2 without battery) / 40 Euro (prototype 3) which is comparable to the one by Lessel et al. (ca. 26 Euro [16]) and the one by Soubam et al. (ca. 22 Euro [22]) since some components are different. The battery lifetime of the prototypes can just roughly be compared since they use different components, but also transmission and power saving strategies. The prototype by Lessel et al. runs for about 31 hours [16], the one by Soubam et al. for around 8 hours [22], but up to 20 hours with different settings. Our prototypes usually run for about 3 weeks permanently, but there is still room for improvement by adjusting software and hardware.

\subsection{Usage of the Scale}

The scale can be used to weigh the portion of food, but also the volume of many fluids as they mostly contain water which has a density of $1.000 \mathrm{~kg} / \mathrm{m}^{3}$. Usually vessels are used to place foodstuff on the scale. Thus, we need to subtract the weight of the vessel to get the amount of the portion. In combination with our app the user can choose out of the most convenient sequence in each situation. The user can decide whether to first weigh the empty and then the full vessel or the other way round. It is also possible to save the weight of empty vessels so that it can directly be selected in the app. Another option we provide is to save a portion of food or drink amount including the type as favorite if the portions are usually about the same. Of course this depends on the type of foodstuff. Although this method is less accurate, this feature helps to reduce the necessity to use the scale every time.

\section{IMPLEMENTATION}

\subsection{Overview}

For the integrated prototype, we used the two Bluetooth scale prototypes, a smartphone (Huawei Nexus 6P) with Android 7.1 and a round smartwatch with "chin" (Motorola Moto 360 gen 1) 
running Android Wear 1.5. In Figure 1, all devices used for the high fidelity prototype including the two Bluetooth scales are shown. The communication between the devices is visible in Figure 2.

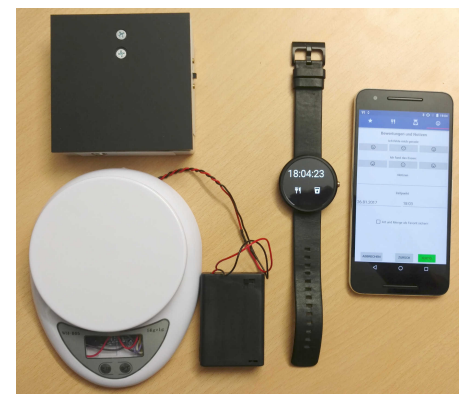

Figure 1: Devices used for the high fidelity system prototype

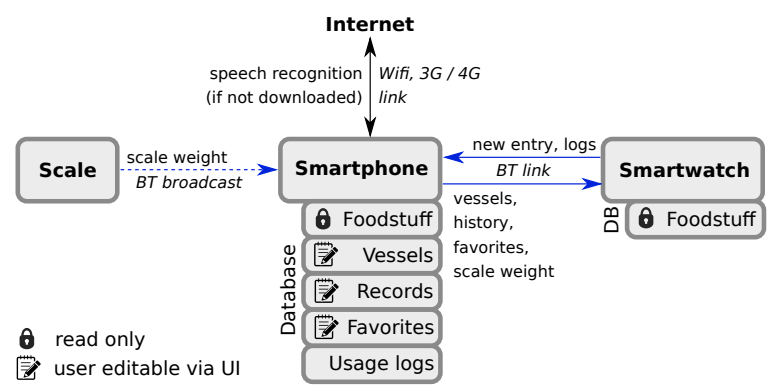

Figure 2: Communication between the devices

Due to their size, smartphones have a much higher battery capacity than smartwatches (for our devices: $3.450 \mathrm{mAh}$ vs. $320 \mathrm{mAh}$ ). Thus, battery consuming tasks like wireless communication should be offloaded to the smartphone. For this reason the connection to the Internet via Wifi is established by the smartphone and it is also listening for the Bluetooth advertisement packets of the scale. Internet is currently just required for Android's speech recognition if the language has not been downloaded. The method for Android's offline speech recognition achieves a word error rate of $13.5 \%$ [19].

To enable continuous interaction between the devices, data synchronization is essential and one of the requirements identified above. The synchronization between the smartscale, smartphone and smartwatch is done by using Bluetooth (BT). The favorites, history (at most 10 entries) and vessel lists are sent to the smartwatch after an update request from the smartwatch has been received. Weight values from the scale received by the smartphone are directly sent to the smartwatch. New entries created by the smartwatch, but also usage logs are transmitted to the smartphone where they are getting stored.

Following the requirements listed above, the smartphone and watch have a local foodstuff database used to simplify the selection of the food and drink type. The database has been populated with German foodstuff names that were gathered from websites on the Internet as there is no free national database. The smartwatch database is reduced to more essential entries (from around 30.000 to about 7.000 entries) since it is quite difficult to search for specific ones on a smartwatch without any text input and speech recognition not always being an option. All data that has to be stored is added to an additional database on the smartphone. All vessels, foodstuff entries (including pictures), favorites and app usage logs are stored here. The table with vessels is populated on first start up with entries which can directly be used, but also edited by the user later on. Also, new vessels can be added.

\subsection{User Interface}

In Figure 3 the most relevant screens related to the recording process of the smartphone and smartwatch app are shown. We will focus in the following on food, but for drinks the process is similar.

The task on both devices can start directly from the main screens of the specific device. This means on the smartphone the home screen where a widget has been placed on the watch, a custom watch face is used which is visible in Figure 1. On both devices, the user can select whether to record food, drink or output the currently measured weight from the scale. Our smartphone prototype additionally allows for viewing and editing already recorded entries by touching a specific entry in the history (see Figure 3). It also enables the supplementation of incomplete entries from the smartwatch later on which are highlighted in orange. In general just type and weight are mandatory to be entered by the user.

On both platforms the user can use the left or right swipe gesture to switch between the tabs. On the smartphone we also provide buttons. Aborting the task is possible by using the Android back button or by pressing "cancel". Due to the lack of space these buttons are not included on the smartwatch GUI. Still, the hardware button of the watch can be pressed and the user can also use the swipe right gesture until the watch face is visible again.

After entering the food record mode, a screen is shown where the user can select items from user-defined favorites or a history of the last 10 different food entries (screen Record 1). This also applies for the smartwatch although favorites are marked with a star and recently used entries (history) with a clock icon. Since there is few space on the smartwatch display, the food entry mode is indicated by the background image. An entry can be selected by touching it. This automatically sets the food type, amount and vessel type / weight. On both devices the last screen Record 4 / Record 5 will be shown after the selection of the favorite / history entry.

If the user decides not to use a preset, he or she can proceed to "Record 2" where the type of food is selected. On the smartphone this is possible by using text input with auto completion (a list with possibly matching entries is shown) and speech recognition using the Google API. Also, a photo of the food can be made by the user or an existing one can be selected from the internal storage. The smartwatch is quite limited in its input capabilities. The entries can just be shown in a list. To make it easier to find a specific entry, presumably rarely used entries were removed from the smartwatch's foodstuff database. The first letter of an entry can be set with an additional list on the left so that the user doesn't have to scroll through the whole list. If the user can't find a specific entry or is in a hurry "later on smartphone" can be chosen and is preset. On the smartphone, the user can edit the type later on, which is possible in a more comfortable way. After that, the user can proceed to Record 3 where the amount can be entered. 


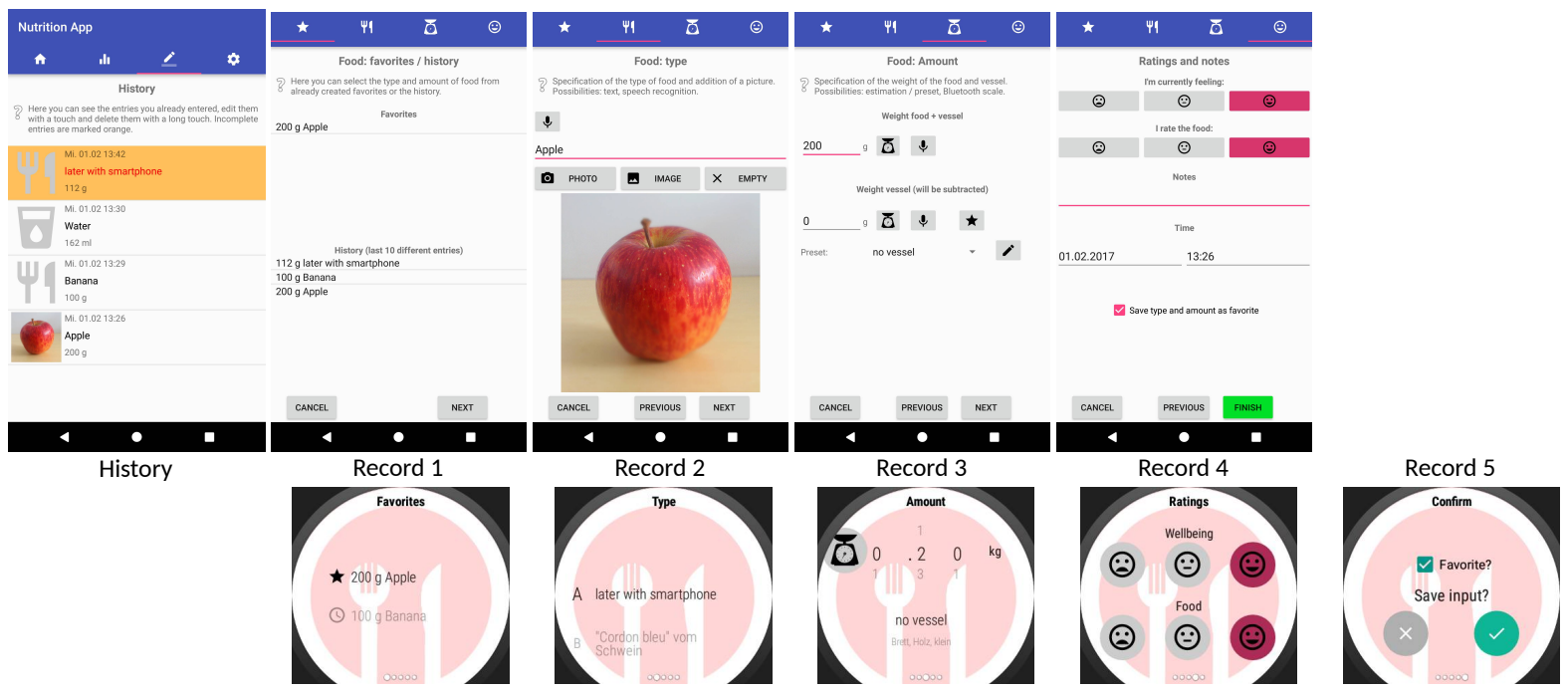

Figure 3: Selected screens of smartphone (top) and smartwatch (bottom)

On both devices the weight of the food including the vessel can be set. The weight can optionally be received from the smartscale. In this case, the user just has to place the food on the scale and press the according button on the smartphone / watch. On the smartphone speech recognition and the on-screen keyboard can additionally be used. The value on the smartwatch can be changed by scrolling through three lists with single digits. The weight of the vessel being subtracted from the total weight can be selected by using a preset list including user defined entries. On the smartphone additionally the weight of the vessel can directly be specified by text input, speech recognition or by using the scale with an empty vessel. The user can additionally directly create a new vessel entry using the current vessel weight (button with "star" icon). A vessel editor is available. On the smartwatch just the selection of an already existing vessel using the list is possible. If the user wants to estimate the weight of the food excluding the vessel or no vessel is used (e.g. a fruit) the default preset can be left unchanged.

On the next screen the overall well-being and the food itself can be rated by the user using a three point scale with smileys. Additionally, on the smartphone custom notes can be entered and the time / date can be changed, which can be useful if the recording is done later on. On the smartwatch just the current system time is used to speed up the process. On the smartwatch the check box allowing to save the entry as favorite is displaced to the confirmation screen (Record 5) to prevent the necessity to scroll vertically.

After pressing the buttons "Finish" or the button with a check symbol the process is finished and the entry is getting stored. If there are missing required fields (type and amount) on the smartphone or the vessel weight is higher than the sum of foodstuff and vessel weight an according information is shown with a message.

\section{EVALUATION}

\subsection{Setup of the Preliminary In-Situ Study}

To evaluate the high fidelity prototype, we gave the prototype for one day to persons that were interested in logging their nutrition intake. We selected people that were already using an Android smartphone with Android 6 or higher since this usually means that they are already familiar in using Android and also the device is recent enough. Several new problems can occur if different devices are used, but it was important that the test persons can use their own devices since it would introduce a different user experience and usage behavior if they had needed to carry an additional smartphone around with them.

We first informed the participants about the procedure and what data would be recorded for the study. Then we presented the prototype. All devices and functions were explained to them. After that we paired the smartwatch with the participant's smartphone. Hereafter the apps for the smartphone and smartwatch were installed. The custom widget and watch face were setup after that. Finally, a short test of the prototype together with the participant was conducted to make the user familiar with the functionality of the prototype. For all participants the smartwatch was a new device which they didn't wear and use before.

The test persons were asked to use the system with their smartphone, the smartwatch and the smartscale for a day to register their food and drink. They could decide on their own where and how to use devices of the prototype. After this day the database with logs and the recorded foodstuff entries were offloaded from the participants' smartphones while they were present.

Additionally, the persons were questioned in a semi-structured interview, containing questions that were included during the gathering of requirements (see section 3). The interviews took about 15 minutes per person and covered 25 questions concerning demographic data, smartphone use, experience with health apps, willingness to use smartwatches, privacy concerns, knowledge on tools for nutrition tracking, motivation, preferred schedule for logging.

\subsection{Semi-Structured Interviews}

In total, we tested the prototype with five participants (females: 1, males: 4) in the age between 24 and 31 (avg. 26.2). Most of them 
were students or working at the university. At first, we obtained demographic data from them as well as information on wearable device usage and nutrition logging.

One of the participants (P5) already logged her fluid intake with a smartphone app ("Plant Nanny") to drink more. All participants rarely used to take pictures of their food, for example, to show them to friends or family members or when they found them exceptionally "aesthetic". Just one person (P1) found that he was able to estimate quite accurately the amount of food and drink. The others thought that they were able to estimate food intake only roughly or food and drink equally inaccurately.

After the general part of the interview, participants were invited to give comments on the nutrition logger and the integrated devices.

Concerning weight estimation, the scale was considered as "an absolute must" to record the amount of food with a sufficient degree of accuracy. P2 thought "the scale was brilliant" since it greatly simplified the weight input. He was also surprised how badly he estimated the weight of food. For example, he did not expect an orange $(235 \mathrm{~g})$ to weigh more than a sandwich he had made $(135 \mathrm{~g})$.

The participants shared the same opinion regarding the usage of smarwatch, smartscale and speech recognition, when it came to upholding privacy.

P5 was concerned about using the scale in public: "It is also strange to weigh in public. If I go to Mc Donald's I wouldn't unpack my scale to place my burger on it." The smartwatch was also appreciated by users who did not want to reveal to others that they had to $\log$ their nutrition. P3 said: "It could be embarrassing if other people find out that you have to track your nutrition." The speech recognition feature for text input was especially appreciated by P2 for usage at home, but he wouldn't use it, for example, at work: "At work, when I'm standing near a colleague, I would feel awkward.” The speech recognizer worked correctly for the words he inputted to $\log$ nutrition. Other participants also thought that the speech recognition could provide a useful addition in private situations. Nevertheless, most of them used it only rarely during the tests.

The context also influenced the preference for one or the other device: P4 said that the smartwatch could be useful when being in a hurry: "If there is no time to input data in detail, entries can be completed later on with the smartphone." Only P1 found the scale little useful since he was mostly on route during the time he tested the prototype. The other participants employed the smartscale at home. They could also imagine using it at work, but not on the go.

Viewed from the point of usability, the multi device setup showed advantages. Participants thought that the smartwatch (in combination with the scale) was in particular useful when repeated food items had to be recorded since in this case the favorites / history could be used. Furthermore, they found the smartwatch convenient in situations where hands were busy or when the smartphone was out of reach. While users relied on the smartwatch when repeated entries had to be made, they preferred the smartphone for inputting new food categories or for providing more details on data that was previously recorded with the smartphone. Overall, the users found the smartphone useful since it included all the necessary functions for food logging.

The participants did not report any problems when having to switch from one device to the other. P2 said that he had noticed that the GUI of the smartwatch had a similar structure as the GUI of the smartphone and that the mapping of GUI elements was clear to him.

According to our logs P1 didn't use the scale with the smartwatch. P2 and P4 employed the scale with the smartwatch and smartphone several times. P3 mentioned that he wanted to make use of the scale in combination with the smartwatch, but at first didn't notice that there was a button to get the values of the scale on the smartwatch. The other participants didn't mention such a problem.

Reminders for nutrition intake logging were requested by P1 and P5. P5 would appreciate if the system reminded her to drink regularly every two / three hours. The other participants didn't mention any problems remembering to log food or drink intake, but rather were concerned that frequent reminders might be disturbing. They mentioned that they would just appreciate reminders on working days (P2) or if they were context-sensitive (P3).

The multi-device nutrition logger helped people reflect on food intake. P2 who was reporting issues with kidney stones said that rating the foodstuff made him "think about whether it tasted good or not”. He also enjoyed taking photos of foodstuff and looking at them later. P2 also expressed strong interest in using the nutrition logger on a long-term basis since he felt the system helped him ensure that he intakes a sufficient amount of fluid.

\section{SUGGESTED EXTENSIONS}

One problem the participants identified was the organization of the large number of foodstuff database entries. As a consequence, the selection of foodstuff was very time-consuming and hardly possible on the smartwatch. P5 mentioned that the foodstuff database was partly inconsistent and confusing since, for example, some entries included a brand name that did not always appear at the beginning. She also mentioned that the auto-completion showed many other foodstuff entries even if she just wanted to insert chocolate as the word is included in several other food entries. In line with her comments, P2 suggested organizing the many entries in a better manner. $\mathrm{P} 1$ and $\mathrm{P} 4$ asked for a possibility to add a meal including multiple ingredients so that the input could be additionally simplified.

Natural language interaction had been requested by P1 as he mentioned that he would like to tell the ingredients of a meal directly to the system while cooking it and having busy hands. This feature does not seem to be included in commercial apps [10] and would be an interesting addition that would have to be researched independently and is also very situation-specific.

\section{DISCUSSION}

Our study showed that the participants can imagine using multiple devices for nutrition tracking and have no problem switching between smartphone and smartwatch. Smartwatches would be accepted as additional device in everyday situations, while the smartscale usage would be mostly limited to private situations e.g. at home. Speech recognition faces the same issue in raising awareness and would be rather used in private spaces.

Non the less smartscales are attested to convey potential to enhance the accuracy of nutrition logging as people can hardly estimate weights without training or the usage of tools like portion size estimation aids. The smartwatch on the other hand raises less 
attention to the act of logging and therefore would encourage the logging in public.

On the topic of reminders for nutrition intake, we think they might also be given in combination with reminders to log nutrition data. Furthermore, we believe that the custom watch face served as a kind of ambient reminder and had a similar function as the stickers used by Kim et al. [15] even though the participants were not aware of it. The interviews indicate, that photos were especially taken of self-made well-prepared dishes. As own cooking is harder to track than ready-to-use food with according labels and takes place mostly at home, a smartscale seems a to be a natural fit here.

\section{CONCLUSION}

In this paper we presented a nutrition logging system that leverages the specific capabilities of multiple devices and modalities for recording nutrition. Our system combines a smartphone, a smartwatch and a smartscale and offers touch- and speech-based input in addition to tangible input by placing objects on the smartscale. This way, our nutrition logging system offers greater flexibility to users than currently available smartscale systems that typically rely on a smartphone only as an additional interaction device. A preliminary in-situ study revealed that users indeed made use of all three devices and exploited the advantages of all of them in a situation-specific manner.

As future work, longer in-situ studies should be conducted so that more situations can occur to enable us to test the usability of specific components of the system in more detail by study participants. There are still several possibilities that could improve the presented system. It is also possible to add a context-aware intelligent reminder which could be implemented using the mobileSSI framework [9] with different modalities. Like Franco et al. [10] already mentioned natural speech processing (NLP) and image recognition could also be a useful addition. A study comparing the different input modalities like it has been done with picture and text input by Lim et al. [17] could also give further insights.

\section{REFERENCES}

[1] O. Amft, H. Junker, and G. Tröster. 2005. Detection of Eating and Drinking Arm Gestures Using Inertial Body-worn Sensors. In Proceedings of the Ninth IEEE International Symposium on Wearable Computers (ISWC '05). IEEE Computer Society, Washington, DC, USA, 160-163. https://doi.org/10.1109/ISWC.2005.17

[2] O. Amft, M. Stäger, P. Lukowicz, and G. Tröster. 2005. Analysis of Chewing Sounds for Dietary Monitoring. UbiComp 2005: Ubiquitous Computing 3660 (2005), 56-72. https://doi.org/10.1007/11551201 4

[3] E. Årsand, M. Muzny, M. Bradway, J. Muzik, and G. Hartvigsen. 2015. Performance of the First Combined Smartwatch and Smartphone Diabetes Diary Application Study. Fournal of Diabetes Science and Technology 9, 3 (2015), 556-563. https: //doi.org/10.1177/1932296814567708 PMID: 25591859.

[4] M. Beigl, H. Gellersen, and A. Schmidt. 2001. Mediacups: experience with design and use of computer-augmented everyday artefacts. Computer Networks 35,4 (2001), 401-409. https://doi.org/10.1016/S1389-1286(00)00180-8

[5] C. M. Carter, J. V. Burley, C. Nykjaer, and E. J. Cade. 2013. Adherence to a Smartphone Application for Weight Loss Compared to Website and Paper Diary: Pilot Randomized Controlled Trial. F Med Internet Res 15, 4 (15 Apr 2013), e32. https://doi.org/10.2196/jmir.2283

[6] F. Cordeiro, E. Bales, E. Cherry, and J. Fogarty. 2015. Rethinking the Mobile Food Journal: Exploring Opportunities for Lightweight Photo-Based Capture. In Proceedings of the 33rd Annual ACM Conference on Human Factors in Computing Systems (CHI '15). ACM, New York, NY, USA, 3207-3216. https://doi.org/10.1145/ 2702123.2702154

[7] F. Cordeiro, D. A. Epstein, E. Thomaz, E. Bales, A. K. Jagannathan, G. D. Abowd, and J. Fogarty. 2015. Barriers and Negative Nudges: Exploring Challenges in Food Journaling. In Proceedings of the 33rd Annual ACM Conference on Human
Factors in Computing Systems (CHI '15). ACM, New York, NY, USA, 1159-1162. https://doi.org/10.1145/2702123.2702155

[8] T. Dong, E. F. Churchill, and J. Nichols. 2016. Understanding the Challenges of Designing and Developing Multi-Device Experiences. In Proceedings of the 2016 ACM Conference on Designing Interactive Systems (DIS '16). ACM, New York, NY, USA, 62-72. https://doi.org/10.1145/2901790.2901851

[9] S. Flutura, J. Wagner, F. Lingenfelser, A. Seiderer, and E. André. 2016. MobileSSI: Asynchronous Fusion for Social Signal Interpretation in the Wild. In Proceedings of the 18th ACM International Conference on Multimodal Interaction (ICMI 2016). ACM, New York, NY, USA, 266-273. https://doi.org/10.1145/2993148.2993164

[10] R. Z. Franco, R. Fallaize, J. A. Lovegrove, and F. Hwang. 2016. Popular NutritionRelated Mobile Apps: A Feature Assessment. FMIR mHealth and uHealth 4, 3 (2016), e85. https://doi.org/10.2196/mhealth.5846

[11] Y. Gao, N. Zhang, H. Wang, X. Ding, X. Ye, G. Chen, and Y. Cao. 2016. iHear Food: Eating Detection Using Commodity Bluetooth Headsets. In Connected Health: Applications, Systems and Engineering Technologies (CHASE), 2016 IEEE First International Conference on. IEEE, 163-172.

[12] W. Jia, H. Chen, Y. Yue, Z. Li, J. Fernstrom, Y. Bai, C. Li, and M. Sun. 2014. Accuracy of food portion size estimation from digital pictures acquired by a chest-worn camera. Public health nutrition 17, 8 (2014), 1671-81. https://doi.org/10.1017/ S1368980013003236

[13] A. Kadomura, C. Li, Y. Chen, K. Tsukada, I. Siio, and H. Chu. 2013. Sensing Fork: Eating Behavior Detection Utensil and Mobile Persuasive Game. In CHI '13 Extended Abstracts on Human Factors in Computing Systems (CHI EA '13). ACM, New York, NY, USA, 1551-1556. https://doi.org/10.1145/2468356.2468634

[14] H. Kalantarian and M. Sarrafzadeh. 2015. Audio-based detection and evaluation of eating behavior using the smartwatch platform. Computers in biology and medicine 65 (2015), 1-9. https://doi.org/10.1016/j.compbiomed.2015.07.013

[15] Y. Kim, S. Ji, H. Lee, J. Kim, S. Yoo, and J. Lee. 2016. "My Doctor is Keeping an Eye on Me!": Exploring the Clinical Applicability of a Mobile Food Logger. In Proceedings of the 2016 CHI Conference on Human Factors in Computing Systems (CHI '16). ACM, New York, NY, USA, 5620-5631. https://doi.org/10.1145/2858036. 2858145

[16] P. Lessel, M. Altmeyer, F. Kerber, M. Barz, C. Leidinger, and A. Krüger. 2016. WaterCoaster: A Device to Encourage People in a Playful Fashion to Reach Their Daily Water Intake Level. In Proceedings of the 2016 CHI Conference Extended Abstracts on Human Factors in Computing Systems (CHI EA '16). ACM, New York, NY, USA, 1813-1820. https://doi.org/10.1145/2851581.2892498

[17] Brian Y Lim, Xinni Chng, and Shengdong Zhao. 2017. Trade-off between Automation and Accuracy in Mobile Photo Recognition Food Logging. In Proceedings of the Fifth International Symposium of Chinese CHI. ACM, 53-59.

[18] R. Lutze, R. Baldauf, and K. Waldhor. 2015. Dehydration prevention and effective support for the elderly by the use of smartwatches. In 2015 17th International Conference on E-health Networking, Application Services (HealthCom). 404-409. https://doi.org/10.1109/HealthCom.2015.7454534

[19] I. McGraw, R. Prabhavalkar, R. Alvarez, M. G. Arenas, K. Rao, D. Rybach, O. Alsharif, H. Sak, A. Gruenstein, F. Beaufays, et al. 2016. Personalized speech recognition on mobile devices. In 2016 IEEE International Conference on Acoustics, Speech and Signal Processing (ICASSP). 5955-5959. https://doi.org/10.1109/ICASSP. 2016.7472820

[20] E. L. Murnane, D. Huffaker, and G. Kossinets. 2015. Mobile health apps. Proceedings of the 2015 ACM International foint Conference on Pervasive and Ubiquitous Computing and Proceedings of the 2015 ACM International Symposium on Wearable Computers - UbiComp '15 (2015), 261-264. https://doi.org/10.1145/2800835. 2800943

[21] H. Sørensen, D. Raptis, J. Kjeldskov, and M. B. Skov. 2014. The 4C Framework: Principles of Interaction in Digital Ecosystems. In Proceedings of the 2014 ACM International foint Conference on Pervasive and Ubiquitous Computing (UbiComp '14). ACM, New York, NY, USA, 87-97. https://doi.org/10.1145/2632048.2636089

[22] S. Soubam, M. Agrawal, and V. Naik. 2017. Using an Arduino and a smartwatch to measure liquid consumed from any container. In 2017 th International Conference on Communication Systems and Networks (COMSNETS). 464-467. https://doi.org/ 10.1109/COMSNETS.2017.7945434

[23] J. Wagner, A. Seiderer, F. Lingenfelser, and E. André. 2015. Combining hierarchical classification with frequency weighting for the recognition of eating conditions. In INTERSPEECH 2015, 16th Annual Conference of the International Speech Communication Association, Dresden, Germany, September 6-10, 2015. 889-893. http://www.isca-speech.org/archive/interspeech_2015/i15_0889.html

[24] G. M. Weiss, J. L. Timko, C. M. Gallagher, K. Yoneda, and A. J. Schreiber. 2016. Smartwatch-based activity recognition: A machine learning approach. 2016 IEEEEMBS International Conference on Biomedical and Health Informatics (BHI) (2016), 426-429. https://doi.org/10.1109/BHI.2016.7455925

[25] B. Zhou, J. Cheng, M. Sundholm, A. Reiss, W. Huang, O. Amft, and P. Lukowicz. 2015. Smart table surface: A novel approach to pervasive dining monitoring. In 2015 IEEE International Conference on Pervasive Computing and Communications (PerCom). 155-162. https://doi.org/10.1109/PERCOM.2015.7146522 